\title{
The Utilization of Context Signals in the Analysis of ABR Potentials by Application of Neural Networks
}

\author{
Andrzej Izworski ${ }^{1}$, Ryszard Tadeusiewicz ${ }^{1}$, and Andrzej Pasławski ${ }^{2}$ \\ ${ }^{1}$ University of Mining and Metallurgy, Dept. of Automatics, Laboratory of Biocybernetics \\ Al. Mickiewicza 30, 30-059 Cracow, Poland \\ \{izwa, rtad)@biocyb.ia.agh.edu.pl \\ ${ }^{2}$ University of Mining and Metallurgy, Dept. of Automatics \\ Al. Mickiewicza 30, 30-059 Cracow, Poland
}

\begin{abstract}
The elaboration of head-surface registration techniques for auditory potentials evoked from the brainstem (ABR) enabled the construction of objective research and diagnostic methods, which can utilized in the examinations of auditory organs. The aim of the present work was the construction of a method, making use of the neural network techniques, enabling an automated detection of wave $\mathrm{V}$ in the ABR signals. The basic problem encountered in any attempts of automated analysis of the auditory potentials is connected with impossibility of a reliable evaluation of a single response evoked by a weak acoustic signal. It has been assumed that considerably better detection results should be obtained, when additional context information will be provided to the network's input. This assumption has been verified using complex, hybrid neural networks. As a result about $90 \%$ of correct recognitions has been achieved
\end{abstract}

\section{Introduction}

The registration and analysis of the ABR (Auditory Brainstem) potentials enables an objective evaluation of functions of both the mechanical part of the auditory system as well as the analysis of processes taking place in the specific levels of the neural part of that system. The registration of the ABR potentials is of particular importance in those cases when the application of the classical audiometric methods is difficult or even impossible.

The typical time dependence of the ABR potential consists of five to seven waves, labeled by the respective roman numbers (I-VII), extracted from the EEG signal by the synchronous averaging and registered within 10 or $12 \mathrm{~ms}$ from the application of the acoustic stimulus. In the medical evaluation of the ABR potential mostly the latency period of wave $\mathrm{V}$ and the $\mathrm{I}-\mathrm{V}$ time distance are taken into account. The absence of any wave, particularly the wave $\mathrm{V}$, is also of great diagnostic importance, and the measurement of the product of the amplitudes of the waves $\mathrm{V}$ and $\mathrm{I}$ is an important indicator used in the evaluation of regularity of processes taking place in the neural part of the auditory system. The above description of forms and methods of processing the ABR potentials in the auditory system is neither complete nor exhaustive, it is however easy to notice that the diagnostic value is mostly represented by the quantities related to the wave $\mathrm{V}$, so its automated detection and localization is an important scientific challenge and a research goal of great practical importance. 


\section{The Research Basis}

The research described in the present work have been carried out mostly in the field of analysis and processing of the ready ABR potential signals registered previously in a clinic. However for introducing the experimental conditions, to which the results described below should be referred, it is necessary to present a few information concerning the applied methodology of inducing and registration of the studied signals.

As it is known, the determination of hearing sensitivity using the ABR potentials consists of the observation of decrease of the amplitude and increase of the latency period for the wave $\mathrm{V}$, for a sequence of $\mathrm{ABR}$ signals registered for stimuli of gradually reduced intensity (e.g. from 110 to $20 \mathrm{~dB}$ with $10 \mathrm{~dB}$ step). The examination goes on till the wave $\mathrm{V}$ totally disappears, what denotes the situation of total lack of signal reception by the patient. The shapes of the reference ABR signals, obtained in the specified conditions for the persons with correct auditory modality, are known (see Fig.1). It is also known that the changes in recording of the ABR signal for persons with the brainstem auditory centers deficiency, consist of deformation of the shape and eventually the disappearance of the wave V.

The basic problem encountered during the attempts of automated analysis of the ABR signals is the fact that the registered signal usually considerably deviates form the reference signal shown in Fig.1.

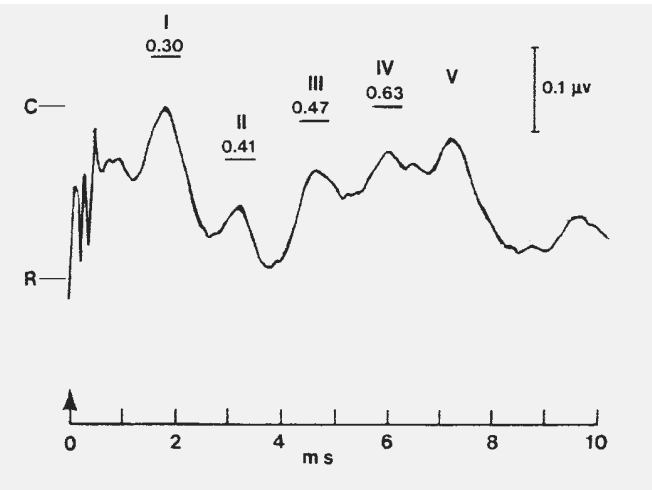

Fig. 1. Typical, singular signal of the ABR

In the case of low levels of the signal stimulating the response distinguishing between particular waves in the ABR recording can be very difficult. The previous works by the authors $[4,5]$ have shown that no algorithm can be constructed, able to perform the task in an automated way, and also that it is extremely difficult to construct and train a neural network, which could be able to determine from evaluation of a single recording of an ABR invoked by a weak auditory signal, the presence or absence of the wave $\mathrm{V}$ in the studied signal and where it is located.

It has been found that better results are obtained by physicians by evaluation of ABR result from the fact, that very often they make use of the context, i.e. evaluating a single run they make use of the neighboring runs. A working hypothesis has been 
formulated, stating that artificial neural networks can also achieve considerably better correctness of the wave $\mathrm{V}$ detection and localization in the recordings of ABR signal, if context information is fed to their inputs, e.g. the ABR signal obtained for the previous (higher) amplitude of the acoustic stimulus.

The research, oriented towards verification of truth of the hypothesis formulated above, have been carried out according to the following research assumptions:

$>$ it was assumed, that although there is a whole set of methods of ABR analysis, the present study will be concentrated exclusively on the attempt of determination whether the wave $\mathrm{V}$ is present or not in a given ABR signal,

$>$ it was assumed that the tool used for detection of the wave $\mathrm{V}$ will be an artificial neural network of the multilayer perceptron structure,

$>$ it was assumed that two signals will be fed to the network's input: the signal of the analyzed ABR and the signal used as a context,

$>$ it was assumed that the data source will be provided by the set of several hundreds of ABR signals registered in the clinical conditions and offered for the present study due to courtesy of the Institute of Control Systems in Katowice.

In accordance to the previous research by the authors [4,5] neural networks have been used for detection of the presence of wave V. The decision followed from the fact, that neural networks are successfully applied for a long time in various, often very diverse areas [1,2]. In papers by other authors $[7,8,9]$ their usefulness has been also proved in the field of medicine.

\section{The Objective of the Study and Way to Achieve It}

In table I selected results are shown of the automated classification of the ABR signals obtained in the previous research stages, oriented towards recognition of isolated signals by the artificial neural networks. The network's input have been fed with a signal describing the analyzed ABR recording (100 points) and the network's output a single logical-type signal was expected, indicating the presence or absence of the wave $\mathrm{V}$ in the input signal. The studied network architectures exhibited the 100-n1 structure (where $n$ denotes the size of the optimized hidden layer) or alternatively 100-n-m-1 structure, for the cases when networks with two hidden layer were applied.

Starting from the observation that a physician undertaking the analysis of recorded ABR signals does not analyze the signals separately but in the context of the accompanying signals (the person sees the whole series of recorded signals obtained for gradually decreasing intensity of the acoustic stimulus), an original technique of the context analysis of the considered signals have been proposed and applied. In the present work for the first time results are shown for a study, in which the authors attempted to take the context into account in the process of classification of $A B R$ signals by the artificial neural networks.

In order to take the context into account in the described study two signals have been fed at the same time to networks input: the presently analyzed ABR signal and the previous, accompanying signal, obtained for the higher intensity of the acoustic stimulus. It could have been achieved using a simple multilayer neural network, but 
additionally attempts have been made to use in the recognition process neural networks of some more complicated architecture.

\section{The Considered Architectures of the Neural Networks Studied}

In the task of automated recognition of the ABR potentials artificial neural networks have been applied, learned by the error backpropagation methods. Two classes of signals have been considered: the class of signals in which wave $\mathrm{V}$ was present and the class of signals where wave V was absent. The simplest architecture (Fig.2), which provides the possibility of making use of the context during the recognition of signals of auditory response is a neural network to the input of which two signals are fed in sequence. The considered architectures effectively exhibited the $200-n-1$ or 200- $n-m-1$ structures.

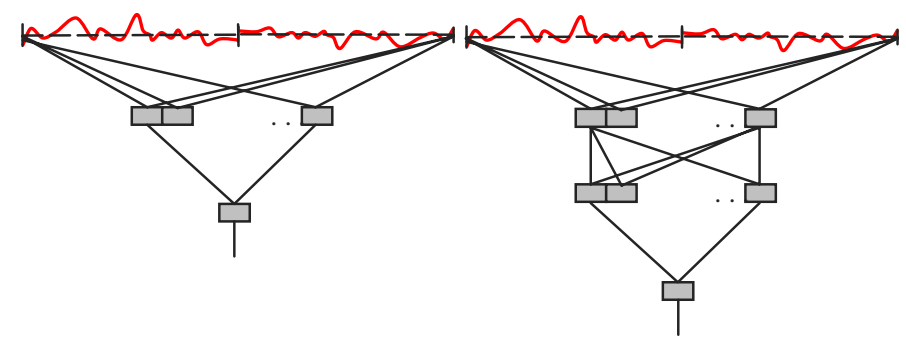

Fig. 2. The double and triple layer neural networks, in which the neurons are connected according to the "every with each other", doublets of signals are fed to the network's input

The network architecture described above have been later modified in such a way, that the first hidden layer has been split into two parts, and then two component signals of the input vector have been fed separately to each of the layers (Fig.3).

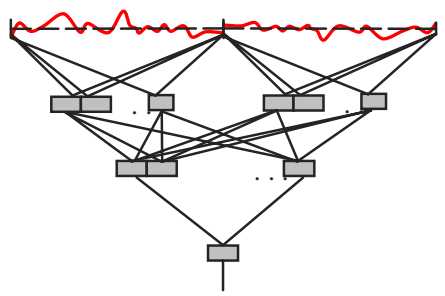

Fig. 3. Triple-layer network, the first hidden layer has been split, so that two consecutive ABR signals are fed separately to the network

Due to such a procedure the split layers of the hidden layer preliminary process the signal to be recognized and its context signal, working independently.

In the following step the first hidden layer can be split into more layers, and the respective signal parts of the two component signals of the input vector should be fed to individual layers. In that case each separated group of neurons can analyze the similarity of different fragments of the signal to be recognized and the context signal. The network of such architecture is presented in Fig. 4. 


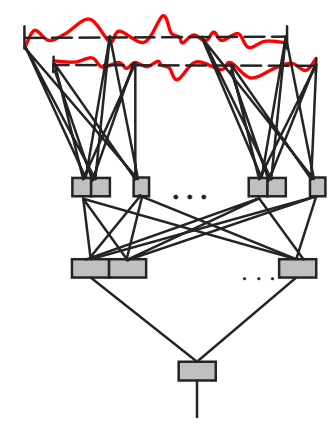

Fig. 4. Triple-layer network, the first hidden layer split into several groups of neurons, to each of them the respective parts of both signals (of equal lengths) are fed

Another possibility is to use only the information of the context signal recognition during the classification of the signal, contrary to the previous case when the whole context signal has been used. In such a situation the context signal should be previously classified by an independent network and then the output signal of that network should be fed to input of the main network together with signal to be classified. Such a network exhibiting a cascade structure is presented in Fig.5.

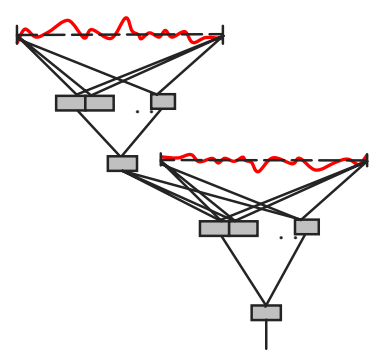

Fig. 5. System consisting of two neural networks, each of them trained separately. The first networks classifies a single signal, and the second network's input is provided with the signal itself and the information about the classification of the context signal

\section{The Data Used for Evaluation of the Utility of Studied Networks}

The data concerning the acquisition techniques for the ABR potentials analyzed in the present work have been as follows: the patient have been applied an acoustic stimulus in the form of a cracking noise of the intensity between 70 and $20 \mathrm{~dB}$, and next from the EEG signal the ABR signal has been extracted. The original size of the signal included 1000 digitally processed values (covering the $10 \mathrm{~ms}$ time period of the signal), but next it was reduced by the proper averaging techniques to 100 values, providing the input data for the considered networks.

The input vectors necessary for the context studies have been constructed in such a way, that to each of the ABR signals has been appended in the front part by the 
preceding signal, obtained in the same measuring sequence but for the higher amplitude of the acoustic stimulus. For studied done using the network presented in Fig.5 the data sets have additionally preprocessed.. The resulting input vectors 192 points long ( 2 x 96 points) have been fed to the network's input.

\section{The Obtained Results}

In the course of the simulation the network's architecture has been optimized in order to provide the best results of the recognition. In table I several best results are shown (for comparison), obtained from the classification of single input signals. These results have already been published.

Table 1. Selected best results of the classification of single signals

\begin{tabular}{|c|c|c|r|r|r|}
\hline \multirow{2}{*}{$\begin{array}{c}\mathrm{N} \\
\mathrm{o}\end{array}$} & \multirow{2}{*}{ NN architecture } & \multirow{2}{*}{ Epochs } & \multirow{2}{*}{$\begin{array}{c}\text { RMS } \\
\text { error }\end{array}$} & & \multicolumn{2}{|c|}{ Error of the classification [\%] } \\
\cline { 5 - 6 } & & & Learning set & \multicolumn{1}{c|}{ Test set } \\
\hline 1 & $100 \times 10 \times 1$ & 271 & 0.899 & 98.68 & 83.12 \\
\hline 2 & $100 \times 8 \times 1$ & 479 & 0.094 & 100.00 & 85.71 \\
\hline 3 & $100 \times 7 \times 2 \times 1$ & 414 & 2.000 & 97.37 & 85.71 \\
\hline 4 & $100 \times 4 \times 4 \times 1$ & 595 & 0.990 & 100.00 & 85.71 \\
\hline
\end{tabular}

On the other hand the tables below the new results for the study of recognition of the ABR signals making use of the context signals.

From the completed research it follows that including the context has the strongest positive influence on the classification quality for the networks including one hidden layer, for which the improvement of the ABR recognition results was about 4-5\%

The conclusion, which can be drawn is that by the addition to the recognized signal only the information about the classification of the signal preceding the analyzed signal leads to much worse effects than including the whole context signal. It has also turned out that application of more complex network architectures of the neural networks does not lead to the increase of ABR signal recognition quality.

Table 2. Selected best results of the classification for the networks presented in Fig. 2 and 3

\begin{tabular}{|c|c|r|r|r|r|}
\hline \multirow{2}{*}{ No } & \multirow{2}{*}{ NN architecture } & \multirow{2}{*}{ Epochs } & \multirow{2}{*}{$\begin{array}{l}\text { RMS } \\
\text { Error }\end{array}$} & & Error of the classification [\%] \\
\cline { 5 - 6 } & & 330 & 1.98 & Learning set & Test set \\
\hline 1 & $200 \times 10 \times 1$ & 395 & 1.24 & 96.05 & 87.01 \\
\hline 2 & $200 \times 8 \times 1$ & 365 & 1.43 & 96.05 & 89.61 \\
\hline 3 & $200 \times 7 \times 2 \times 1$ & 1049 & 3.94 & 97.37 & 88.31 \\
\hline 4 & 2080 & 3.87 & 94.74 & 85.71 \\
\hline 5 & $(100+100) \times(4+4) \times 2 \times 1$ & 1794 & 3.88 & 94.74 & 87.01 \\
\hline 6 & $(100+100) \times(3+3) \times 2 \times 1$ & 2250 & 3.87 & 94.74 & 87.01 \\
\hline 7 & $(100+100) \times(3+3) \times 2 \times 1$ & & 94.74 & 88.31 \\
\hline
\end{tabular}


Table 3. Selected best result of the classification for the networks shown in Fig.4

\begin{tabular}{|c|c|r|c|r|r|}
\hline \multirow{2}{*}{ No } & \multirow{2}{*}{ NN architecture } & \multirow{2}{*}{ epochs } & \multirow{2}{*}{ RMS error } & \multicolumn{2}{|c|}{$\begin{array}{r}\text { Error of the classification } \\
{[\%]}\end{array}$} \\
\cline { 5 - 6 } & & & & Learning set & Test set \\
\hline 1 & $101 \times 10 \times 1$ & 1094 & 1.02 & 98.68 & 84.42 \\
\hline 2 & $101 \times 10 \times 1$ & 392 & 1.127 & 98.68 & 83.12 \\
\hline 3 & $101 \times 8 \times 1$ & 462 & 0.198 & 100.00 & 83.12 \\
\hline
\end{tabular}

Table 4. Selected best results of the classification for the network shown in Fig.5

\begin{tabular}{|c|c|r|r|r|r|}
\hline \multirow{2}{*}{ No } & NN architecture & \multirow{2}{*}{ epochs } & \multirow{2}{*}{$\begin{array}{l}\text { RMS } \\
\text { error }\end{array}$} & \multicolumn{2}{|c|}{$\begin{array}{c}\text { Error of the } \\
\text { classification [\%] }\end{array}$} \\
\cline { 5 - 7 } & & & & Learning set & Test set \\
\hline 1 & $(192) \times(3+3+3+3) \times 3 \times 1$ & 886 & 1.98 & 97.37 & 80.52 \\
\hline 2 & $(192) \times(4+4+4+4) \times 3 \times 1$ & 702 & 4.88 & 96.05 & 84.42 \\
\hline 3 & $(192) \times(3+3+3+3) \times 4 \times 1$ & 595 & 0.99 & 100.00 & 85.71 \\
\hline
\end{tabular}

\section{Conclusion}

Summarizing the above considerations it can be concluded that the application of the input signal including the context of the analyzed ABR signal considerably improves the network's ability for recognition of the presence (or absence) of the wave $\mathrm{V}$ in the analyzed signal. It has been also found that increasing the network's complexity (by transition from triple layer to quadruple layer networks) does not lead to the expected improvement in the recognition quality, while considerably increasing the duration of the learning process. Neither have the expected results been obtained by the attempted optimization of the network's operation by splitting the hidden layer into two part analyzing separately the recognized signal and its context signal. The attempt to improve the network's operation by comparing the respective signal parts has not lead to satisfactory results either.

In spite of those - partly negative - results, it can be stated, that the application of the context signal was the reason that the considered task has found a more satisfactory solution, comparing to the case when the context was not taken into account. The neural network making use of the context data, which after the learning process were the best in classification of the ABR signals, have obtained the correct recognition in $88-89 \%$ cases. This result has to be regarded as satisfactory. It shown, that it is possible to build an automated system based on the neural networks, detecting (with the satisfactory recognition reliability) the presence of wave $\mathrm{V}$ in the ABR signal. It is the most important result of the study described here.

At the same time it was shown, that making use of the context signal during the automated recognition of ABR signals by artificial neural network is meaningful and leads to an effect observed as the improvement of the classification quality. This is the most essential cognitive effect of the described study. 


\section{References}

1. Tadeusiewicz R.: Neural Networks, AOW RM, Warszawa (1993), (in polish)

2. Żurada J., Barski M., Jędruch W.: Artificial Neural Networks, PWN, Warszawa (1996), (in polish)

3. Aminoff M.J. (ed.): Elektrodiagnosis in Clinical Neurology, Churchil Livingstone New York, (1986)

4. Pasławski A., Izworski A.: Detection of the V wave in BAEP with use of artificial neural networks, III Conference on Acoustical Methods and Biomedical Engineering, Zakopane (1998), (in polish)

5. Izworski A.: Analysis of the Brainstem Auditory Evoked Potentials with Use Neural Networks, X Conference „Biocybernetics and Biomedical Engineering”, Warszawa, (1997) 808-813, (in polish)

6. Delgado R. E., Ozdmar O.: Automated Auditory Brainstem Response Interpretation, IEEE Engineering In Medicine And Biology, April/May (1994)

7. Tadeusiewicz R., Wszołek W., Izworski A., Modrzejewski M.: Application of Neural Networks in Diagnosis of Pathological Speech, In M. Heiss (ed.): Proceedings of the International ICSC/IFAC Symposium on Neural Computation NC '98, Vienna University of Technology, ICSC Academic Press, Canada/Switzerland, (1998) 1040-1045

8. Tadeusiewicz R., Wszołek W., Izworski A., Wszołek T.: Methods of deformed speech analysis, In: Manfredi C., Bruscaglioni P.: Models and Analysis of Vocal Emissions for Biomedical Applications, University of Firenze, (1999) 130-137

9. Tadeusiewicz R., Izworski A., Wszołek W., Wszołek T.: Processing and Classification of Deformed Speech Using Neural Networks, in: Fouke J.M., Nerem R.M., Blanchard S.M., Yoganathan A.P., (eds.): Serving Humanity, Advancing Technology, Proceedings of The First Joint BMES/EMBS Conference, Atlanta, (1999) 927-928 